\title{
Structure of bird assemblages in dry and seasonally flooded grasslands in Itirapina Ecological Station, São Paulo state
}

\author{
Fieker, CZ. ${ }^{a *}$, Reis, $M$ G. $^{a}$ and Dias Filho, MM. ${ }^{b}$ \\ aPós-graduação em Ecologia e Recursos Naturais, Universidade Federal de São Carlos - UFSCar, \\ Rod. Washington Luís, Km 235, CEP 13565-905, São Carlos, SP, Brazil \\ ${ }^{b}$ Departamento de Ecologia e Biologia Evolutiva, Universidade Federal de São Carlos - UFSCar, \\ Rod. Washington Luís, Km 235, CEP 13565-905, São Carlos, SP, Brazil \\ *e-mail: carolfieker@gmail.com \\ Received December 6, 2011 - Accepted March 22, 2012 - Distributed February 28, 2013
}

(With 1 figure)

\begin{abstract}
Grasslands are characteristic physiognomies of the Brazilian Cerrado domain. One of last remnants of these threatened environments in the state of São Paulo is located in Itirapina Ecological Station, considered as an Important Bird Area (IBA). We investigated bird assemblages that use seasonally flooded grasslands, and predominantly dry grasslands, from August 2010 until July 2011. We focused on aspects of assemblage structure, and possible responses of birds to environmental changes. We recorded 76 species, of which $23.7 \%$ are threatened. Some basic aspects of assemblages were similar between environments, such as diversity descriptors, and the tendency of increase richness in the same periods. Other parameters differed between environments, such as absolute richness, recorded monthly (lower in seasonally flooded grasslands). Considering time dimension in analyses, we observed differences in assemblage structure over time, within and between environments. When birds were grouped in guilds, assemblage structure did also differ. We identified significant correlations between the abundance of some guilds and environmental variables. Changes in the availability of resources and microhabitats may affect structure and organization of the studied bird assemblages.
\end{abstract}

Keywords: avifauna, grasslands, microhabitat, food resource, seasonality.

\section{Estrutura das assembleias de aves em campos limpos secos e sazonalmente alagáveis na Estação Ecológica de Itirapina, estado de São Paulo}

\section{Resumo}

Os campos são fisionomias características do domínio do Cerrado no Brasil. Um dos últimos remanescentes destes ambientes ameaçados no Estado de São Paulo está localizado na Estação Ecológica de Itirapina, a qual é considerada uma IBA (Important Bird Area). Foram investigadas as assembleias de aves que utilizaram os campos limpos sazonalmente alagáveis e os campos limpos predominantemente secos, de agosto de 2010 a julho de 2011 . Neste estudo, focaramse aspectos da estrutura das assembleias e possíveis respostas das aves às mudanças ambientais. Foram registradas 76 espécies, das quais 23,7\% estão ameaçadas. Alguns aspectos básicos das assembleias foram similares entre os ambientes, como os descritores de diversidade e a tendência de aumento da riqueza durante os mesmos períodos; outros aspectos se distinguiram, como a riqueza absoluta registrada mensalmente (menor nos campos limpos sazonalmente alagáveis). Ao considerar-se a dimensão temporal nas análises, notam-se diferenças na estrutura ao longo do tempo, dentro e entre os ambientes. Quando as aves foram agrupadas em guildas, a estrutura das assembleias também se mostrou diferente. Identificaram-se correlações significativas entre a abundância de algumas guildas e as variáveis ambientais. Mudanças na disponibilidade de recursos e micro-habitats podem afetar a estrutura e a organização das assembleias das aves estudadas.

Palavras-chave: avifauna, campo limpo, micro-habitat, recurso alimentar, sazonalidade.

\section{Introduction}

Some characteristics of natural environments and their habitats, such as weather conditions, resources availability, and interactions between biotic components, may act as filters that can determine which species of the regional pool can occur in a local scale (Keddy, 1992; Cornwell et al., 2006). These factors frequently change over time and space, and their variations may affect ecological processes at the individual, population, and community levels (Begon et al., 2006; Yang et al., 2008; Flesch and Steidl, 2010).

Seasonal climatic variations, such as increases in rainfall, may create pulses of resource availability that allow new species to colonise the habitat, or that lead to an 
increase in the abundance of resident species (Yang et al., 2008). Chesson et al. (2004) stated that temporal variation in rainfall creates 'temporary niche opportunities'. In ecosystems marked by intense climatic seasonality, such as the Brazilian Cerrado, environmental changes can be frequently observed on a large scale (Sarmiento, 1983; Ratter et al., 1997; Becerra et al., 2009).

The Cerrado is the second largest phytogeographical and morphoclimatic domain of South America (Ab'Saber, 1977; Scariot et al., 2005), and it is considered as the most threatened and biodiverse savanna in the world (Klink and Machado, 2005). The Cerrado has been pointed out by Myers et al. (2000) as one of the 25 biodiversity hotspots of the world with conservation priorities. It is composed of forest, savanna, and grassland formations, each one with distinct physiognomies (Coutinho, 1978; Ribeiro and Walter, 1998; Batalha, 2011). Historically, grassland ecosystems have been always among the most threatened formations in many regions of the world (Filgueiras, 2002; Alho, 2005), mainly because their structure facilitates occupancy and land use (Stotz et al., 1996; Bond and Parr, 2010).

In São Paulo state, most bird species that inhabit grassland environments survive only in Itirapina Ecological Station. It is the most important Conservation Unit of the state for the conservation of Cerrado birds (São Paulo, 2009), and the only IBA (Important Bird Area) not located in the coastal region, according to Bencke et al. (2006).

Hence, the present study aims at describing and identifying changes in structure aspects (composition and abundance of species and guilds) of bird assemblages that inhabit two distinct environments of the "campo limpo' grasslands in Itirapina Ecological Station. We also investigated possible correlations between the presence and abundance of bird guilds, and the seasonal availability of resources and microhabitats.

\section{Material and Methods}

\subsection{Study area}

The Itirapina Ecological Station (IES), located within the municipalities of Brotas and Itirapina, São Paulo state $\left(22^{\circ} 11^{\prime} \mathrm{S}-22^{\circ} 15^{\prime} \mathrm{S}\right.$ and $\left.47^{\circ} 51^{\prime} \mathrm{W}-47^{\circ} 57^{\prime} \mathrm{W}\right)$, cover an area of 2,300 ha. It harbours all vegetation physiognomies of the 'cerrado sensu lato' gradient (according to the classification of Coutinho, 1978), and also other natural formations, such as gallery forests and permanently wet savannas. This reserve protects one of the largest fragments of grasslands ('campo limpo'), and open savanna physiognomies ('campo sujo' and 'campo cerrado') of the Cerrado domain in the state of São Paulo (Bencke et al., 2006).

The regional climate is Cwa according to Köppen (1948) classification, characterised by well-defined dry (April to September) and rainy (October to March) seasons (Brasileiro et al., 2005; Zanchetta et al., 2006). During the rainy season, substrates of the lower portions of the topographic gradient, or that are closer to groundwater reservoirs, are subject to water saturation, which may result in the formation of temporary ponds and marshes. On the other hand, in the dry season, the sandy soil (predominantly Quartzarenic Neosol) undergoes a severe and progressive drought until the subsequent rainy season (Silva, 2005; Tannus et al., 2006).

The ecosystems of interest in the present study were grasslands with no shrub-tree stratum, where herbaceous plants are predominant, mainly grasses. There may be some isolated and very rare shrubs in some locations that do not alter the characteristics of these environments. Grasslands cover nearly 160 ha of IES, and an adjacent protected area. Inside grasslands, two different environments are found: predominantly dry grasslands and seasonally flooded grasslands. Despite an apparent similarity in structure between these environments, reinforced by the spatial continuum of the grasses, there are floristic differences. Seasonally flooded grasslands seem to be dominated by grasses of the genus Andropogon L., especially A. bicornis L. and A. leucostachyus Kunth, whereas dry grasslands have higher herbaceous plant richness, and more even species distribution (pers. obs.). The aforementioned grass species were recorded by Tannus (2007) in IES, where the substrate varies from humid to saturated.

\subsection{Procedures}

We defined sites for the bird survey, from March 2010 to July 2010, based on field screening of the largest grassland patches of IES. Three almost circular buffer sites (approximately $3 \mathrm{ha}$ ) of homogeneous landscape were defined in each environment studied: the seasonally flooded grasslands (FloGr), and the predominantly dry grasslands (DryGr). Then we collected data systematically over 12 months, from August 2010 to July 2011.

Each site was sampled monthly. Bird records were carried out in a delimited circular area (50 m radius, $\pm 10 \mathrm{~m}$ error, within an area of approximately $0.8 \mathrm{ha}$ ), located in a central zone of each 3 ha buffer, in order to avoid influences of other environments. Buffer sites were located at least $200 \mathrm{~m}$ away from each other, and no sampling site was located at less than $100 \mathrm{~m}$ from other physiognomies. We recorded birds that used microhabitats in the delimited area early in the morning (from sunrise to10:00), or in the late afternoon (from 16:00 until sunset), at fixed intervals of 15 minutes. Sound records were considered as belonging to a single individual, even for species that live in groups, except when more than one vocalization was perceived at the same time. We used binoculars, digital recorders (when sound identification was necessary), and GPS devices for initial mapping, georeferencing, and to find the sampling sites. The scientific and vernacular names of bird species followed the Brazilian Ornithological Records Committee (CBRO, 2011).

For characterization and monitoring of environmental changes, we set up monthly 20 plots of $2 \times 2 \mathrm{~m}$ in each environment, randomly distributed within the three buffer areas. We selected six variables to be monitored in the field: (1) herbaceous stratum cover; (2) exposed soil cover; 
(3) presence of superficial water; (4) presence of amphibian eggs; (5) presence of tadpoles; and (6) presence of insect larvae. The variables 1 and 2 were visually estimated in percentage for each plot, but the final percentage values for each environment were calculated as the mean of all 20 plots sampled monthly. For the other four variables, we recorded presence/absence in each plot to calculate the total percentage of presence for all plots. Regional monthly rainfall data (in $\mathrm{mm}$ ) were the same for both environments, and were obtained from the meteorological station of Centro de Recursos Hídricos e Ecologia Aplicada (CRHEA-EESC/USP), located approximately $2 \mathrm{~km}$ away from the northern limit of IES.

Bird guild definition followed the original concept, which was widely discussed by Simberloff and Dayan (1991). The concept is based on species grouping according to resource use, without considering taxonomic relationships. Guilds were based on foraging microhabitats and food preferences of each species. Foraging microhabitats were determined from the literature (Stotz et al., 1996; Sick, 1997; Sigrist, 2006, 2009) and field observations. The dietary classification of the species followed Motta-Junior (1990), Sick (1997), and Sigrist (2006, 2009), and was complemented with field records.

\subsection{Data analysis}

All data, sets and treatments had their normality tested with Shapiro-Wilk and Lilliefors tests, calculated in BioEstat (Ayres et al., 2007). Parametric tests were used, whenever data normality was confirmed.

Scores of Shannon diversity (H') were compared with a specific t-test (Hammer et al., 2001; Magurran, 2004). Other indexes of species composition and abundance, such as dominance (D) and equitability (J) (Zar, 1999; Magurran, 2004), were compared by bootstrapping (Hammer et al., 2001). Species composition and guilds were compared between environments with a multivariate analysis (NPMANOVA) with Bonferroni correction (Manly, 2008), which assesses changes in biological communities over time or along environmental gradients (Clarke, 1993; Hammer et al., 2001). The contribution of each guild to changes between environments over time was tested with SIMPER (Similarity Percentage). This multivariate method calculates the overall average dissimilarity among treatments and samples, and its overall significance was assessed with an ANOSIM procedure, using the Bray-Curtis similarity (Clarke, 1993). The SIMPER method calculated also each species' contribution to the differences observed between and within samples. These analyses were run in the program PAST (Hammer et al., 2001).

We compared variations in species richness between environments and over time, with a two-way ANOVA. In addition, a post hoc t-test was used to compare means. We used a PCA to find the most relevant environmental variables, i.e., indicated by this analysis as the main components of environmental data. Correlations were made using Pearson's coefficient. We removed correlations derived from datasets with less than four samples. When there were multiple significant correlations, we discarded the weakest ones, which generally deviate from the biological information found in the literature. These analyses were performed in BioEstat (Ayres et al., 2007).

\section{Results}

We recorded 76 bird species in the sampling sites; 18 are threatened and 15 are highly dependent on grassland environments (Table 1). In the predominantly dry grassland (DryGr) we recorded 56 species, and in the seasonally flooded grassland (FloGr) 58 species. We found 38 species (50\%) present in both environments, whereas $20(26.3 \%)$ were recorded exclusively in FloGr, and 18 (23.7\%) in DryGr.

There was no significant difference between environments in diversity indexes of bird assemblages, considering the whole dataset (DryGr H' = 3.04; FloGr H' = 3.02; $\mathrm{t}=0.231, \mathrm{df}=1877.5, \mathrm{P}=0.816)$. The other community descriptors, such as dominance (DryGr D $=0.090$; FloGr $\mathrm{D}=0.085$; bootstrap $\mathrm{P}=0.207$ ) and equitability (DryGr $\mathrm{J}=0.762$; FloGr $\mathrm{J}=0.752$; bootstrap $\mathrm{P}=0.425)$, have also no significant differences between environments.

However, qualitative temporal data showed that the bird assemblages of the two environments were different $(\mathrm{F}=2.694, \mathrm{P}=0.002)$. After testing for temporal differences between bird assemblages, we investigated which structural aspects were responsible for the seasonal changes. Rainfall was considered as the most relevant environmental variable for the temporal characterization of samples, and it may also have caused some changes observed in other environmental variables (e.g., ponds appearing after rainy days). Rainfall was pointed out as the main component of environmental variables in the PCA, and as an important variable for both areas, responsible for $88.23 \%$ of the variation recorded in DryGr, and $85.83 \%$ in FloGr. Rainfall was also positively correlated with other environmental variables in one or both environments (superficial water: DryGr $\mathrm{R}=0.605$, $\mathrm{R}^{2}=0.366, \mathrm{P}=0.037$; FloGr $\mathrm{R}=0.819, \mathrm{R}^{2}=0.672$, $\mathrm{P}=0.001$; insect larvae: DryGr $=\mathrm{ns}$; FloGr $\mathrm{R}=0.789$, $\mathrm{R}^{2}=0.623, \mathrm{P}=0.002$; amphibian eggs: $\mathrm{DryGr}=\mathrm{ns} ;$ FloGr $\mathrm{R}=0.746, \mathrm{R}^{2}=0.556, \mathrm{P}=0.005$; and tadpoles: DryGr $=\mathrm{ns}$; FloGr R = 0.917, $\left.\mathrm{R}^{2}=0.841, \mathrm{P}<0.0001\right)$.

There was no significant correlation between diversity ( $\mathrm{H}^{\prime}$ ) and rainfall in both environments (DryGr $\mathrm{R}=0.476$, $\mathrm{R}^{2}=0.226, \mathrm{P}=0.117$; FloGr $\mathrm{R}=0.263, \mathrm{R}^{2}=0.069$, $\mathrm{P}=0.408$ ). But the analysis of richness data indicated that the number of observed species per month in FloGr was positively correlated with rainfall, though a similar trend was not observed in DryGr (DryGr $\mathrm{R}=0.473, \mathrm{R}^{2}=0.224$, $\mathrm{P}=0.12$; FloGr $\mathrm{R}=0.688, \mathrm{R}^{2}=0.472, \mathrm{P}=0.013$ ). Nevertheless, monthly richness of both areas were positively correlated $\left(\mathrm{R}=0.778, \mathrm{R}^{2}=0.606, \mathrm{P}=0.003\right)$.

The variance of bird species richness differed between environments $(\mathrm{F}=12.898, \mathrm{P}=0.0003$; i.e., the number of recorded species per month was not the same between environments), and throughout months $(\mathrm{F}=7.613, \mathrm{P}=0.0015$; i.e., there was monthly variation in number of species in each of the two environments). A post hoc test pointed out that average of bird records differed between environments 
Table 1. List of bird species recorded in dry (DryGr) and seasonally flooded (FloGr) campo limpo grasslands of Itirapina Ecological Station. Values represent species' relative abundance in each environment. Guild acronyms are given in Table 2. Species conservation status followed the red list of São Paulo state (SPRL) (2009), and IUCN's red list (IUCN, 2011), NT: near threatened, VU: vulnerable, EN: endangered, CR: critically endangered. Endemism in the Cerrado domain (EC) followed Silva (1997), Cavalcanti (1999), and Silva and Bates (2002).

\begin{tabular}{|c|c|c|c|c|}
\hline Family/species & Guilds & DryGr & FloGr & $\begin{array}{c}\text { Status SPRL/ } \\
\text { IUCN }\end{array}$ \\
\hline \multicolumn{5}{|l|}{ Rheidae } \\
\hline Rhea americana (Linnaeus, 1758) * & T-Omn & 0.003 & - & $\mathrm{CR} / \mathrm{NT}$ \\
\hline \multicolumn{5}{|l|}{ Tinamidae } \\
\hline Crypturellus parvirostris (Wagler, 1827) * & $\mathrm{T}-\mathrm{Omn}$ & 0.004 & - & - \\
\hline Rhynchotus rufescens (Temminck, 1815) ** & $\mathrm{T}-\mathrm{Omn}$ & 0.016 & 0.008 & VU \\
\hline Nothura maculosa (Temminck, 1815) ** & T-Omn & 0.002 & - & - \\
\hline \multicolumn{5}{|l|}{ Anhimidae } \\
\hline Anhima cornuta (Linnaeus, 1766) & AqT-PhytOmn & - & 0.002 & $\mathrm{CR}$ \\
\hline \multicolumn{5}{|l|}{ Anatidae } \\
\hline Dendrocygna viduata (Linnaeus, 1766) & AqT- PhytOmn & - & 0.05 & - \\
\hline Amazonetta brasiliensis (Gmelin, 1789) & AqT- PhytOmn & 0.002 & 0.218 & - \\
\hline \multicolumn{5}{|l|}{ Ciconiidae } \\
\hline Mycteria americana Linnaeus, $1758 * \mathrm{M}$ & AqT-Car & - & 0.019 & NT \\
\hline \multicolumn{5}{|l|}{ Ardeidae } \\
\hline Tigrisoma lineatum (Boddaert, 1783) & AqT-Car & - & 0.001 & - \\
\hline Ardea cocoi Linnaeus, 1766 & AqT-Car & - & 0.004 & - \\
\hline Ardea alba Linnaeus, 1758 & AqT-Car & - & 0.01 & - \\
\hline Syrigma sibilatrix $($ Temminck, 1824$) *$ & AqT-InsCar & - & 0.019 & - \\
\hline Egretta thula (Molina, 1782) & AqT-InsCar & - & 0.004 & - \\
\hline \multicolumn{5}{|l|}{ Threskiornithidae } \\
\hline Theristicus caudatus (Boddaert, 1783) * & AqT-InsCar & - & 0.021 & - \\
\hline \multicolumn{5}{|l|}{ Cathartidae } \\
\hline Coragyps atratus (Bechstein, 1793) * & T-Det & - & 0.002 & - \\
\hline \multicolumn{5}{|l|}{ Falconidae } \\
\hline Caracara plancus (Miller, 1777) * & T-CarDet & - & 0.003 & - \\
\hline Milvago chimachima (Vieillot, 1816) * & T-CarDet & 0.001 & - & - \\
\hline \multicolumn{5}{|l|}{ Rallidae } \\
\hline Micropygia schomburgkii (Schomburgk, 1848) * & T-Ins & 0.001 & - & $\mathrm{CR}$ \\
\hline Aramides cajanea (Statius Muller, 1776) & $\mathrm{T}-\mathrm{Omn}$ & - & 0.001 & - \\
\hline Amaurolimnas concolor (Gosse, 1847) & $\mathrm{T}-\mathrm{Omn}$ & - & 0.001 & - \\
\hline Porzana albicollis (Vieillot, 1819) * & $\mathrm{T}-\mathrm{Omn}$ & 0.027 & 0.031 & - \\
\hline Pardirallus nigricans (Vieillot, 1819) & AqT-Omn & - & 0.003 & - \\
\hline \multicolumn{5}{|l|}{ Cariamidae } \\
\hline Cariama cristata (Linnaeus, 1766) * & T-InsCar & 0.014 & 0.005 & - \\
\hline \multicolumn{5}{|l|}{ Charadriidae } \\
\hline Vanellus chilensis (Molina, 1782) ** & T-InsCar & - & 0.041 & - \\
\hline \multicolumn{5}{|l|}{ Jacanidae } \\
\hline Jacana jacana (Linnaeus, 1766) & AqT-Omn & - & 0.01 & - \\
\hline \multicolumn{5}{|l|}{ Columbidae } \\
\hline Patagioenas picazuro (Temminck, 1813) * & T-Gra & - & 0.003 & - \\
\hline Zenaida auriculata (Des Murs, 1847) * & T-Gra & 0.015 & - & - \\
\hline
\end{tabular}

Level of dependence on grasslands formations: (*) birds that inhabit grasslands optionally (Vickery et al., 1999; Bagno and Marinho-Filho, 2001) and (**) birds that are strongly associated with grasslands habitats (Vickery et al., 1999; Bagno and Marinho-Filho, 2001; São Paulo, 2009). Migratory movements (M) followed Sick (1997), Motta-Junior et al. (2008) and Willis (2004). 
Table 1. Continued...

\begin{tabular}{|c|c|c|c|c|}
\hline Family/species & Guilds & DryGr & FloGr & $\begin{array}{l}\text { Status SPRL/ } \\
\text { IUCN } \\
\end{array}$ \\
\hline \multicolumn{5}{|l|}{ Cuculidae } \\
\hline Tapera naevia (Linnaeus, 1766) * & HerT-InsCar & 0.001 & - & - \\
\hline \multicolumn{5}{|l|}{ Strigidae } \\
\hline Athene cunicularia (Molina, 1782) ** & T-InsCar & - & 0.011 & - \\
\hline \multicolumn{5}{|l|}{ Trochilidae } \\
\hline Eupetomena macroura (Gmelin, 1788) & H-NecIns & 0.002 & 0.002 & - \\
\hline Colibri serrirostris (Vieillot, 1816) & H-NecIns & 0.002 & 0.002 & - \\
\hline Chlorostilbon lucidus (Shaw, 1812) & H-NecIns & 0.001 & - & - \\
\hline Hylocharis chrysura (Shaw, 1812) & H-NecIns & 0.003 & - & - \\
\hline \multicolumn{5}{|l|}{ Picidae } \\
\hline Colaptes campestris (Vieillot, 1818) * & T-Ins & 0.021 & 0.002 & - \\
\hline \multicolumn{5}{|l|}{ Melanopareiidae } \\
\hline Melanopareia torquata $(\mathrm{Wied}, 1831) *$ & HerT-Ins & 0.021 & 0.008 & $\mathrm{EN}(\mathrm{EC})$ \\
\hline \multicolumn{5}{|l|}{ Furnariidae } \\
\hline Synallaxis frontalis Pelzeln, 1859 & H-Ins & 0.001 & - & - \\
\hline Synallaxis albescens Temminck, $1823 *$ & H-Ins & 0.027 & 0.009 & NT \\
\hline Synallaxis spixi Sclater, 1856 & H-Ins & 0.004 & 0.002 & - \\
\hline \multicolumn{5}{|l|}{ Rynchocyclidae } \\
\hline $\begin{array}{l}\text { Hemitriccus margaritaceiventer } \\
\text { (d'Orbigny \& Lafresnaye, 1837) }\end{array}$ & H-Ins & 0.001 & - & - \\
\hline \multicolumn{5}{|l|}{ Tyrannidae } \\
\hline Camptostoma obsoletum (Temminck, 1824) & H-Ins & 0.003 & - & - \\
\hline Elaenia flavogaster (Thunberg, 1822) & HerAer-Ins & 0.001 & - & \\
\hline Elaenia chiriquensis Lawrence, $1865 * \mathrm{M}$ & HerAer-Ins & 0.001 & 0.002 & - \\
\hline Culicivora caudacuta (Vieillot, 1818)** & H-Ins & 0.005 & 0.004 & $\mathrm{CR} / \mathrm{VU}$ \\
\hline Polystictus pectoralis (Vieillot, 1817)** & H-Ins & 0.005 & 0.003 & $\mathrm{CR} / \mathrm{NT}$ \\
\hline Serpophaga subcristata (Vieillot, 1817) & H-Ins & 0.001 & 0.001 & - \\
\hline Tyrannus savana Vieillot, $1808 * \mathrm{M}$ & HerAer-Ins & 0.008 & 0.001 & - \\
\hline Myiophobus fasciatus (Statius Muller, 1776) & H-Ins & 0.002 & - & - \\
\hline Gubernetes yetapa (Vieillot, 1818)* & HerAer-Ins & 0.014 & 0.024 & - \\
\hline Alectrurus tricolor (Vieillot, 1816)**M & H-Ins & 0.016 & 0.004 & $\mathrm{CR} / \mathrm{VU}$ \\
\hline Xolmis cinereus (Vieillot, 1816) *M & H-Ins & 0.004 & 0.003 & - \\
\hline Xolmis velatus (Lichtenstein, 1823)*M & H-Ins & - & 0.001 & - \\
\hline \multicolumn{5}{|l|}{ Hirundinidae } \\
\hline Pygochelidon cyanoleuca (Vieillot, 1817) * & Aer-Ins & 0.009 & - & - \\
\hline Alopochelidon fucata (Temminck, 1822) * & Aer-Ins & 0.014 & 0.015 & - \\
\hline Stelgidopteryx ruficollis (Vieillot, 1817) *M & Aer-Ins & 0.02 & 0.009 & - \\
\hline Tachycineta leucorrhoa (Vieillot, 1817) * & Aer-Ins & 0.003 & - & - \\
\hline \multicolumn{5}{|l|}{ Troglodytidae } \\
\hline Troglodytes musculus Naumann, 1823 & H-Ins & 0.001 & 0.001 & - \\
\hline \multicolumn{5}{|l|}{ Mimidae } \\
\hline Mimus saturninus (Lichtenstein, 1823) & HerT-InsOmn & 0.01 & 0.001 & - \\
\hline
\end{tabular}

Level of dependence on grasslands formations: (*) birds that inhabit grasslands optionally (Vickery et al., 1999; Bagno and Marinho-Filho, 2001) and (**) birds that are strongly associated with grasslands habitats (Vickery et al., 1999; Bagno and Marinho-Filho, 2001; São Paulo, 2009). Migratory movements (M) followed Sick (1997), Motta-Junior et al. (2008) and Willis (2004). 
Table 1. Continued...

\begin{tabular}{|c|c|c|c|c|}
\hline Family/species & Guilds & DryGr & FloGr & $\begin{array}{c}\text { Status SPRL/ } \\
\text { IUCN } \\
\end{array}$ \\
\hline \multicolumn{5}{|l|}{ Motacillidae } \\
\hline Anthus lutescens Pucheran, $1855 * *$ & T-Ins & 0.004 & 0.002 & - \\
\hline \multicolumn{5}{|l|}{ Thraupidae } \\
\hline Saltatricula atricollis (Vieillot, 1817) * & HerT-Gra/Ins & 0.053 & 0.01 & VU (EC) \\
\hline Cypsnagra hirundinacea (Lesson, 1831) * & $\mathrm{H}-\mathrm{InsOmn}$ & 0.004 & - & EN (EC) \\
\hline Neothraupis fasciata (Lichtenstein, 1823) * & HerT-InsOmn & 0.022 & 0.007 & $\mathrm{EN} / \mathrm{NT}(\mathrm{EC})$ \\
\hline \multicolumn{5}{|l|}{ Emberizidae } \\
\hline Zonotrichia capensis (Statius Muller, 1776) * & HerT-Gra/Ins & 0.009 & 0.001 & - \\
\hline Ammodramus humeralis (Bosc, 1792) ** & HerT-Gra/Ins & 0.069 & 0.053 & - \\
\hline Sicalis citrina Pelzeln, $1870 *$ & HerT-Gra & 0.022 & 0.008 & NT \\
\hline Sicalis luteola (Sparrman, 1789) $* * \mathrm{M}$ & HerT-Gra & 0.245 & 0.108 & - \\
\hline Emberizoides herbicola (Vieillot, 1817) ** & HerT-Gra/Ins & 0.082 & 0.084 & - \\
\hline Emberizoides ypiranganus Ihering\&Ihering, $1907 * *$ & H-GraIns & 0.007 & 0.005 & VU \\
\hline Volatinia jacarina (Linnaeus, 1766) * & HerT-Gra & 0.086 & 0.055 & - \\
\hline Sporophila plumbea (Wied, 1830) **M & H-Gra & 0.01 & 0.002 & EN \\
\hline Sporophila caerulescens (Vieillot, 1823) ${ }^{\mathrm{M}}$ & H-Gra & 0.055 & 0.012 & - \\
\hline Sporophila leucoptera (Vieillot, 1817) *м & H-Gra & 0.002 & 0.005 & - \\
\hline Sporophila bouvreuil (Statius Muller, 1776) **M & H-Gra & 0.012 & 0.006 & VU \\
\hline Sporophila palustris (Barrows, 1883) **M & H-Gra & - & 0.001 & $\mathrm{CR} / \mathrm{EN}$ \\
\hline \multicolumn{5}{|l|}{ Parulidae } \\
\hline Geothlypis aequinoctialis (Gmelin, 1789) & H-Ins & 0.007 & 0.007 & - \\
\hline \multicolumn{5}{|l|}{ Icteridae } \\
\hline Pseudoleistes guirahuro (Vieillot, 1819) ** & HerT-Gra/Ins & 0.016 & 0.067 & - \\
\hline Molothrus bonariensis (Gmelin, 1789) * & HerT-Gra/Ins & 0.007 & - & - \\
\hline
\end{tabular}

Level of dependence on grasslands formations: (*) birds that inhabit grasslands optionally (Vickery et al., 1999; Bagno and Marinho-Filho, 2001) and (**) birds that are strongly associated with grasslands habitats (Vickery et al., 1999; Bagno and Marinho-Filho, 2001; São Paulo, 2009). Migratory movements (M) followed Sick (1997), Motta-Junior et al. (2008) and Willis (2004).

( $\mathrm{T}=3.591, \mathrm{P}=0.0042$ ), and were lower in FloGr. Bird species richness and rainfall variations are presented in Figure 1.

The 76 bird species recorded belonged to 22 ecological guilds. The DryGr harbored 18 guilds, whereas 20 guilds were present in FloGr. The number of species per guild (with acronyms), and environment are presented in Table 2.

There were significant differences in guild structure between environments throughout the year $(\mathrm{F}=2.781$, $\mathrm{P}=0.0038$ ). The average dissimilarity of guilds between environments over time, in terms of species composition and number of individuals, was $52.96 \%$. This value was proportionally divided among guilds, indicating the contribution of each guild to the total variation (Table 2). Finally, we searched for relationships between environmental variables, and guild presence and abundance. Significant results, showed in Table 3, may be explained by trophic relationships or habitat preferences, and most of them were observed in FloGr areas.

\section{Discussion}

Almost one third (32.9\%) of the 231 bird species of Itirapina Ecological Station (IES) listed by MottaJunior et al. (2008) were found in the predominantly dry grasslands and the seasonally flooded grasslands sampled in the present study. Although grasslands are only one of the physiognomies of IES, they harbour a large number of species. Hence, we can infer that these areas are very important for the local avifauna. In addition, many of these species (18 birds, $23.7 \%$ of the species recorded in our study) are threatened according to IUCN's red list (IUCN, 2011) or the red list of São Paulo State (2009). Moreover, 15 species (19.74\%) are strongly associated with grasslands, and four species are endemic to the Cerrado domain (see Table 1). According to Motta-Junior et al. (2008), the high number of bird species of conservation interest in this Conservation Unit is presumably related to the predominance of grasslands. This situation points to the need for preserving those habitats in order to maintain the 


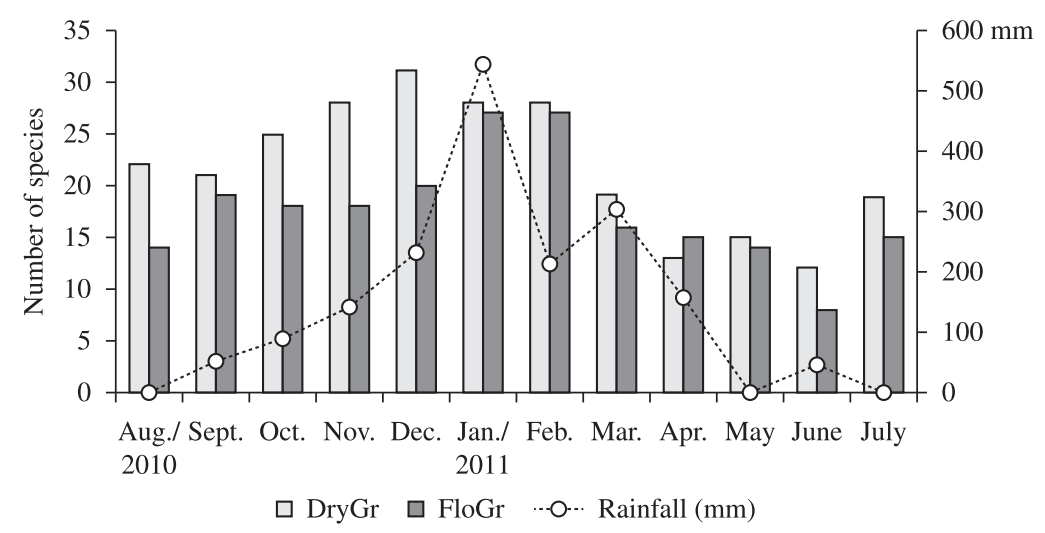

Figure 1. Species richness in dry grasslands (DryGr), in seasonally flooded grasslands (FloGr), and monthly rainfall.

Table 2. Guilds and their respective number of species: total number, number of species exclusive to each environment, and species common to both environments. Guild acronyms used in the text and in other tables are presented here. Values in the last column indicate the contribution of each guild to the overall dissimilarity measured (52.96\%) in the SIMPER analysis.

\begin{tabular}{|c|c|c|c|c|c|c|c|}
\hline \multirow{3}{*}{ Foraging Habitat } & \multirow{3}{*}{ Feeding habit } & \multirow{3}{*}{ Acronyms } & \multicolumn{4}{|c|}{ Number of species } & \multirow{3}{*}{ SIMPER } \\
\hline & & & \multirow{2}{*}{ Total } & \multicolumn{3}{|c|}{ Per environment } & \\
\hline & & & & DryGr & FloGr & Both & \\
\hline \multirow{4}{*}{$\begin{array}{l}\text { Aquatic-Terrestrial } \\
\text { Humid }\end{array}$} & carnivorous & AqT-Car & 4 & 0 & 4 & 0 & 1.36 \\
\hline & phytophagous/omnivorous & AqT-PhytOmn & 3 & 1 & 3 & 1 & 7.46 \\
\hline & insectivorous/carnivorous & AqT-InsCar & 3 & 0 & 3 & 0 & 2.01 \\
\hline & omnivorous & AqT-Omn & 2 & 0 & 2 & 0 & 0.62 \\
\hline \multirow[t]{6}{*}{ Terrestrial } & carnivorous/detritivorous & T-CarDet & 2 & 1 & 1 & 0 & 0.23 \\
\hline & detritivorous & T-Det & 1 & 0 & 1 & 0 & 0.15 \\
\hline & granivorous & T-Gra & 2 & 1 & 1 & 0 & 0.86 \\
\hline & insectivorous & T-Ins & 3 & 3 & 2 & 2 & 1.36 \\
\hline & insectivorous/carnivorous & T-InsCar & 3 & 1 & 3 & 1 & 2.35 \\
\hline & omnivorous & T-Omn & 7 & 5 & 4 & 2 & 1.89 \\
\hline \multirow[t]{5}{*}{ Herbaceous-Terrestrial } & granivorous & HerT-Gra & 3 & 3 & 3 & 3 & 13.93 \\
\hline & granivorous/insectivorous & HerT-Gra/Ins & 6 & 6 & 5 & 5 & 5.78 \\
\hline & insectivorous & HerT-Ins & 1 & 1 & 1 & 1 & 1.08 \\
\hline & insectivorous/carnivorous & HerT-InsCar & 1 & 1 & 0 & 0 & 0.06 \\
\hline & insectivorous/omnivorous & HerT-InsOmn & 2 & 2 & 2 & 2 & 1.92 \\
\hline \multirow[t]{5}{*}{ Herbaceous } & granivorous & H-Gra & 5 & 4 & 5 & 4 & 3.65 \\
\hline & granivorous/insectivorous & H-GraIns & 1 & 1 & 1 & 1 & 0.46 \\
\hline & insectivorous & H-Ins & 14 & 13 & 10 & 9 & 3.17 \\
\hline & insectivorous/omnivorous & H-InsOmn & 1 & 1 & 0 & 0 & 0.26 \\
\hline & nectarivorous/insectivorous & H-NecIns & 4 & 4 & 2 & 2 & 0.57 \\
\hline Herbaceous-Aerial & insectivorous & HerAer-Ins & 4 & 4 & 3 & 3 & 1.18 \\
\hline Aerial & insectivorous & Aer-Ins & 4 & 4 & 2 & 2 & 2.61 \\
\hline
\end{tabular}

regional species pool. Furthermore, there is an aggravating factor that hinders the preservation of threatened and endemic birds: grasslands are endangered both in Brazil and the whole world (Vickery et al., 1999; Olmos, 2005; Bond and Parr, 2010). In São Paulo state, where $95 \%$ of the natural grassland areas have been destroyed in the past three decades (Bencke et al., 2006), IES is one of the few protected remnants that shelter these habitats (Zanchetta et al., 2006).

Despite superficial similarities of these environments as landscape elements, mainly due to the continuum of herbaceous plants, the studied grasslands (FloGr and DryGr) differ in some characteristics. When their structure is investigated on a small scale, there are differences 
Table 3. Significant correlations. Guild acronyms are given in Table 2.

\begin{tabular}{ccccc}
\hline \multicolumn{2}{c}{ Environment/environmental variables } & & Guilds & Correlation results \\
\hline DryGr & Herbaceous cover & $\mathrm{x}$ & HerT-InsOmn & $\mathrm{r}=0.66 ; \mathrm{R}^{2}=0.43 ; \mathrm{P}=0.019$ \\
& & $\mathrm{x}$ & H-Ins & $\mathrm{r}=0.59 ; \mathrm{R}^{2}=0.35 ; \mathrm{P}=0.04$ \\
DryGr & Exposed soil & $\mathrm{x}$ & T-Omn & $\mathrm{r}=-0.58 ; \mathrm{R}^{2}=0.34 ; \mathrm{P}=0.047$ \\
FloGr & Superficial water & $\mathrm{x}$ & AqT-Car & $\mathrm{r}=0.60 ; \mathrm{R}^{2}=0.40 ; \mathrm{P}=0.027$ \\
& & $\mathrm{x}$ & AqT-InsCar & $\mathrm{r}=0.63 ; \mathrm{R}^{2}=0.40 ; \mathrm{P}=0.027$ \\
& $\mathrm{x}$ & AqT-Omn & $\mathrm{r}=0.74 ; \mathrm{R}^{2}=0.40 ; \mathrm{P}=0.027$ \\
& $\mathrm{x}$ & T-InsCar & $\mathrm{r}=0.65 ; \mathrm{R}^{2}=0.40 ; \mathrm{P}=0.027$ \\
& & $\mathrm{x}-$ Thn & $\mathrm{r}=0.61 ; \mathrm{R}^{2}=0.40 ; \mathrm{P}=0.027$ \\
FloGr & Amphibian eggs & $\mathrm{x}$ & T-Omn & $\mathrm{r}=0.65 ; \mathrm{R}^{2}=0.42 ; \mathrm{P}<0.022$ \\
FloGr & Tadpoles & $\mathrm{x}$ & AqT-InsCar & $\mathrm{r}=0.59 ; \mathrm{R}^{2}=0.35 ; \mathrm{P}<0.04$ \\
FloGr & Insect larvae & $\mathrm{x}$ & AqT-PhytOmn & $\mathrm{r}=0.69 ; \mathrm{R}^{2}=0.47 ; \mathrm{P}<0.013$ \\
& & $\mathrm{x}$ & AqT-InsCar & $\mathrm{r}=0.62 ; \mathrm{R}^{2}=0.39 ; \mathrm{P}<0.029$ \\
& & $\mathrm{x}$ & AqT-Omn & $\mathrm{r}=0.72 ; \mathrm{R}^{2}=0.52 ; \mathrm{P}<0.008$ \\
& & $\mathrm{x}$ & T-InsCar & $\mathrm{r}=0.71 ; \mathrm{R}^{2}=0.50 ; \mathrm{P}<0.01$ \\
\hline
\end{tabular}

in micro-habitats (e.g., exposed soil, grass cover) and resource availability (e.g., tadpoles, insect larvae). Thus, assuming that these areas are structurally different, we should also expect differences in bird diversity. However, no differences were found between environments in terms of diversity, dominance, and equitability. These community descriptors (H', D, and E) consider only the number of species (richness) and their abundance (Zar, 1999; Magurran, 2004), thus each species is represented by a single value. In case of species turnover between environments, and throughout the year in each environment, it would be measured as a simple replacement, changing one value for another that could be equal or very similar. These procedures do not allow identifying qualitative changes. Therefore, these diversity indexes allowed only a limited assessment of similarities between assemblages, in terms of the proportion of abundant, intermediate, and rare species. The non-significant correlation between $\mathrm{H}^{\prime}$ and monthly rainfall in the two environments, and the positive correlation in H' between DryGr and FloGr, corroborate the hypothesis of diversity similarity, since both environments exhibited the same trend of variation in H' over time.

Species turnover occurred throughout the year in both environments, but it was more evident in FloGr. Seasonality is a remarkable feature of Neotropical savannas (Sarmiento, 1983). Seasonal variations in climate, mainly those related to rainfall, were followed by changes in bird assemblage structure. This fact was also observed in plant communities of IES by Tannus (2007), who found significant correlations between rainfall and cover percentage, budding, flowering, and senescence of plant species. He concluded that there are large variations in grasslands physiognomies between dry and rainy periods.

In the present study, the correlation between species richness in FloGr and rainfall can be explained, at least in part, by the emergence of some microhabitats such as seasonal ponds, which can temporarily harbor species with life requirements related to aquatic environments (e.g., water dependent or semi-dependent birds, see Table 1 and Table 2). There was no significant relationship between rainfall and bird richness in DryGr, probably because richness increased three months before the beginning of the rainy season (from November 2010 to April 2011, Figure 1). The arrival of migratory birds (e.g., Tyrannus savana, Stelgidopteryx ruficollis) in August and September may have contributed to the increase in richness. However, some birds species considered as migratory (Motta-Junior et al., 2008) have individuals that remain in IES even during the driest months. We recorded some migratory birds (e.g., Elaenia chiriquensis, Alectrurus tricolor) in winter (dry season) in the studied areas, which have also been recorded by Reis (2011) in previous years in the same reserve.

The results revealed differences in richness between environments and over time. It indicates that assemblages may respond to environmental changes differently throughout the year (i.e., turnover of species or guilds), but they remain similar in terms of abundance (i.e., proportion or relative abundance of species or guilds). A significant difference in the average monthly richness in DryGr and FloGr was also found. Unexpectedly, FloGr had the lowest average richness, though it had two exclusive species more than DryGr. This shows that species turnover through time may be an important process that characterizes the structure of grassland bird assemblages, especially in flooded areas.

As suggested in the literature, the structure of assemblages or communities can be better understood if assessed at higher organization levels (Brooks et al., 1998), such as guilds, whose functional responses to environmental changes are more evident (Croonquist and Brooks, 1991; O'Connel et al., 2000). Significant correlations between guilds and environmental variables may not necessarily evidence a direct relationship, as they could happen by chance when analysing multiple correlations. Thus, we have tried to base the discussion on the comparison of our results and data from the literature. 
Aquatic bird guilds, recorded in seasonal ponds of FloGr areas, were positively correlated with insect larvae (insectivorous-carnivorous, omnivorous-phytophagous, and omnivorous), and tadpoles (insectivorous-carnivorous). Insect larvae and tadpoles, as well as their respective adults, may be consumed by birds that belong to these guilds (Moojen et al., 1941; Telino Junior et al., 2003). There are records in the literature of anuran predation by birds (Crump and Vaira, 1991; Johansson, 2008; Silva and Giaretta, 2008). Some bird species can eat even poisonous anurans, for instance birds of the family Ardeidae (Egrets and Herons), and the Buff-necked Ibis Theristicus caudatus (Sick, 1997; França et al., 2004). Many records also pointed out that the emergence of aquatic insects influences the presence and abundance of several birds, since these insects are an important resource for them (Gray, 1993; Power and Rainey, 2000; Murakami and Nakano, 2002; Iwata et al., 2003; Langcore et al., 2006). According to Sick (1997), these arthropods are essential for aquatic birds, e.g., those of the family Anatidae (Ducks), especially during the breeding period. Additionally, insect larvae may be a resource used by terrestrial insectivorous-carnivorous birds; we also found a correlation between them.

Superficial water in temporary ponds was used by birds as a resource and habitat, sometimes simultaneously. The use of this microhabitat may be also affected by the presence and abundance of other resources (e.g., bird prey). The availability of amphibian eggs, tadpoles, and insect larvae was positively correlated with monthly rainfall. Brasileiro et al. (2005) emphasized the relationship between amphibian presence and the rainy season in IES. According to these authors, the breeding period of these animals is generally restricted to the rainy season, except for a single species that breeds throughout the year. Several anuran species recorded by Brasileiro et al. (2005) in IES breed in temporary ponds formed in seasonally flooded grasslands. These microhabitats are a concentrated, but ephemeral, food source for some bird guilds.

Birds that forage in herbaceous/terrestrial microhabitats and that have insectivorous-omnivorous diet, such as strict insectivorous birds of the herbaceous stratum, had a positive correlation with grass cover in DryGr areas, their foraging microhabitat. Terrestrial and omnivorous birds were correlated with amphibian eggs in FloGr areas, probably because they may be an occasional food for some of these birds.

Besides the structural importance of the studied microhabitats as part of vegetation, it is important to notice that they are also a resource to birds, because when in use they become unavailable to other individuals (Alcock, 2005). Ricklefs and Hainsworth (1968) showed that birds may depend on particular microhabitats not only for foraging, but also for thermoregulation in response to high temperatures and insolation. Both the herbaceous cover (mostly grasses) and the presence of superficial water can act as limiting factors for choosing a given area in detriment of others (e.g., areas with exposed soil and no shadow), due to the favorable conditions for some species or guilds in some periods of the annual rain cycle, and even during the daily cycle. For example, in IES the Collared Crescentchest (Melanopareia torquata) was recorded using ground burrows and galleries made by mammals probably as a refuge against climatic conditions of the region, where the daily temperature ranges from $13{ }^{\circ} \mathrm{C}$ to $20{ }^{\circ} \mathrm{C}$, on average (Kanegae and Reis, 2011).

When temporary ponds were formed in FloGr, a transitional community (sensu Ricklefs, 2003) was structured. We noted a succession of bird species and variations in their abundance, according to the water level of these ponds and their resource availability. These communities may be considered as 'discrete communities', which exist only when the necessary conditions for their maintenance are met (Wilbur, 1997). Additionally, we observed tradeoffs in bird assemblages. Some aquatic guilds increased their richness, whereas others experienced a reduction, or even disappeared (e.g., the Burrowing Owl Athene cunicularia, a terrestrial insectivorous bird, disappeared when ponds were formed). Aquatic birds remained in FloGr areas only during the flooding period, such as the Wood Stork (Mycteria americana), recorded for the first time in IES, and observed only in temporary ponds. A single aquatic bird species, the Brazilian Teal (Amazonetta brasiliensis), was recorded in DryGr areas. Insectivores of the herbaceous stratum had their abundance reduced in FloGr areas, such as the Cock-tailed Tyrant (Alectrurus tricolor), and the Pale-breasted Spinetail (Synallaxis albescens). Some rare and threatened species, like the Greater Rhea (Rhea americana), and the Ocellated Crake (Micropygia schomburgkii), appeared only in DryGr areas, whereas other species, such as the Horned Screamer (Anhima cornuta), and the Marsh Seedeater (Sporophila palustris), used only FloGr areas.

In the present study we have showed that seasonality, evidenced by changes in environmental variables (conditions and resources), may influence bird assemblage organization over time. The seasonally flooded and the predominantly dry grasslands of Itirapina Ecological Station shelter bird assemblages with many species of high ecological and conservation value. Since the area is an important remnant of the natural grasslands in the southern Cerrado domain, its conservation relevance is even higher to the local and regional bird species pool, especially to threatened species.

Acknowledgements - We thank CAPES and CNPq for financial support; José Roberto Maramarque from the Centro de Recursos Hídricos e Educação Ambiental (CRHEA-USP) for providing meteorological data; the Instituto Florestal for access permission to study in natural areas; and the staff of Itirapina Ecological and Experimental Stations for assistance during field work. We also thank Mercival Roberto Francisco, Nivaldo Nordi, and the two anonymous referees for helpful comments and suggestions.

\section{References}

AB'SABER, AN., 1977. Os domínios morfoclimáticos na América do Sul: primeira aproximação. Geomorfologia, vol. 53, p. 1-23.

ALCOCK, J., 2005. Animal behavior: an evolutionary approach. 8th ed. Sunderland: Sinauer.

ALHO, CJR., 2005. Desafios para a conservação do Cerrado face às atuais tendências de uso e ocupação. In SCARIOTI, A., SOUSA-SILVA, JC. and FELFILI, JM. (Eds.). Cerrado: 
Ecologia, Biodiversidade e Conservação. Brasília: Ministério do Meio Ambiente.

AYRES, M., AYRES JR., M., AYRES, DL. and SANTOS, AS., 2007. BioEstat 5.0: aplicações estatísticas nas áreas das ciências biomédicas. Sociedade Civil de Mamirauá, CNPq.

BAGNO, MA. and MARINHO-FILHO, J., 2001. A avifauna do Distrito Federal: uso de ambientes abertos e florestais e ameaças. In RIBEIRO, JF., FONSECA, CEL. and SOUSA-SILVA, JC. (Eds.). Cerrado: caracterização e recuperação de Matas de Galeria. Platina: Embrapa. p. 495-528.

BATALHA, MA. 2011. O cerrado não é um bioma. Biota Neotropica, vol. 11 , no. 1, p. 1-4

BECERRA, JAB., SHIMABUKURO, YD. and ALVALÁ, RCS., 2009. Relação do padrão sazonal da vegetação com a precipitação na região de Cerrado da Amazônia legal, usando índices espectrais de vegetação. Revista Brasileira de Meteorologia, vol. 24 , no. 2 , p. $125-134$

BEGON, M., TOWNSEND, CR. and HARPER, JL., 2006. Ecology: from individuals to ecosystems. 4th d. Oxford: Blackwell Publishing.

BENCKE, GA., MAURICIO, GN., DEVELEY, PF. and GOERCK, JM., 2006. Áreas Importantes para a Conservação das Aves no Brasil. Parte I - Estados do Domínio da Mata Atlântica. Editora SAVE Brasil.

BOND, JW. and PARR, CL., 2010. Beyond the forest edge: Ecology, diversity and conservation of the grassy biomes. Biological Conservation, vol. 143, p. 2395-2404. http://dx.doi. org/10.1016/j.biocon.2009.12.012

BRASILEIRO, CA., SAWAYA, RJ., KIEFER, MC. and MARTINS, M., 2005. Anfíbios de um fragmento de Cerrado aberto do sudeste do Brasil. Biota Neotropica, vol. 5, no. 2, p. 1-17.

BROOKS, RP., O'CONNELL TJ., WARDROP, DH. and JACKSON, LE., 1998. Towards a regional index of biological integrity: the example of forested riparian systems. Environmental Monitoring and Assessment, vol. 51, p. 131-143. http://dx.doi. org/10.1023/A:1005962613904

CAVALCANTI, RB., 1999. Bird species richness and conservation in the cerrado region of central Brazil. Studies in Avian Biology, vol.19, p. 244-249.

Comitê Brasileiro de Registros Ornitológicos - CBRO, 2011. Listas das aves do Brasil. Available from: <http://www.cbro.org. br>. Access in: 10 Sept. 2010)

CHESSON, P., GEBAUER, RLE., SCHWINNING, S., HUNTLY, N., WIEGAND, K.., ERNEST, MSK., SHER, A., NOVOPLANSKY, A. and WELTZIN, JF., 2004. Resource pulses, species interactions, and diversity maintenance in arid and semi-arid environments. Oecologia, vol. 141, p. 236-253. http://dx.doi.org/10.1007/ s00442-004-1551-1

CLARKE, KR., 1993. Non-parametric multivariate analysis of changes in community structure. Australian Journal of Ecology, vol. 18, p. 117-143. http://dx.doi.org/10.1111/j.1442-9993.1993. tb00438.x

COUTINHO, LM., 1978. O conceito de cerrado. Revista Brasileira de Botânica, vol. 1, no. 1, p. 17-23.

CORNWELL, WK., SCHWILK, DW. and ACKERLY, DD., 2006. Trait-based test for habitat filtering: convex hull volume. Ecology, vol. 87, p. 1465-1471. http://dx.doi.org/10.1890/00129658(2006)87[1465:ATTFHF]2.0.CO;2
CROONQUIST, MJ. and BROOKS, RP., 1991. Use of avian and mammalian guilds as indicators of cumulative impacts in riparian-wetland areas. Environmental Management, vol. 15, p. 701-714. http://dx.doi.org/10.1007/BF02589628

CRUMP, ML. and VAIRA, M., 1991. Vulnerability of Pleurodema Borelli tadpoles to an avian predator: effect of body size and density. Herpetologica, vol. 47, no. 3, p. 316-321.

FILGUEIRAS, TS., 2002. Herbaceous Plant Communities. In OLIVEIRA, PS. and MARQUIS, RJ. (Eds.). The Cerrados of Brazil: Ecology and Natural History of a Neotropical Savanna. New York: Columbia University Press.

FLESCH, AD. and STEIDL, RJ., 2010. Importance of environmental and spatial gradients on patterns and consequences of resource selection. Ecological Applications, vol. 20, no. 4, p. 1021-1039. http://dx.doi.org/10.1890/09-0076.1

FRANÇA, LF., FACURE, KG., and GIARETTA, AA. 2004. Trophic and special niche of two large sized species of Leptodactylus (Anura) in southeastern Brazil. Studies of Neotropical Fauna And Environment, vol. 39, p. 243-248. http://dx.doi.org/10.1080/01650520400007330

GRAY, LJ., 1993. Response of insectivorous birds to emerging aquatic insects in riparian habitats of a tallgrass prairie stream. American Midland Naturalist, vol. 129, p. 288-300. http://dx.doi. org/10.2307/2426510

HAMMER, Ø., HARPER, DAT. and RYAN, PD., 2001. PAST: Paleontological Statistics Software Package for Education and Data Analysis. Palaeontologia Electronica, vol. 4, no. 1, p. 9. Available from: <http://palaeoelectronica.org/2001_1/past/ issue1_01.htm>. Access in: 28 June 2011.

International Union for Conservation of Nature - IUCN, 2011. IUCN Red List of Threatened Species. version: 2011.1. Available from: <http://www.iucnredlist.org >. Access in: 20 Sept. 2011.

IWATA, T., NAKANO, S. and MURAKAMI, M., 2003. Stream meanders increase insectivorous bird abundance in riparian deciduous forests. Ecography, vol. 26, p. 325-337. http://dx.doi. org/10.1034/j.1600-0587.2003.03355.x

JOHANSSON, P. 2008. Marshes and swamps: a wetland web of life. United State: Library of Congress Cataloging-in-Publication Data.

KANEGAE, MF. and REIS, MG., 2011. Use of burrows by Melanopareia torquata, Collared Crescentchest (Melanopareiidae) in the Cerrado of southeastern Brazil. Revista Brasileira de Ornitologia $=$ Ararajuba, vol. 19, no. 3, p. 398-401.

KEDDY, PA.,1992. Assembly and response rules: two goals for predictive community ecology. Journal of Vegetation Science, vol. 3, no. 2, p. 157-164. http://dx.doi.org/10.2307/3235676

KLINK, CA. and MACHADO, RB., 2005. Conservation of the Brazilian Cerrado. Conservation Biology, vol. 19, p. 707-713. http://dx.doi.org/10.1111/j.1523-1739.2005.00702.x

KÖPPEN, W., 1948. Climatologia. Mexico: Fondo de Cultura Económica.

LANGCORE, JR., McAULEY, DG., PENDELTON, GW., BENNATTI, CR., MINGO, TM. and STROMBORG, KL., 2006. Macroinvertebrate abundance, water chemistry, and wetland characteristics affect use of wetlands by avian species in Maine. Hidrobiologia, vol. 567, p. 143-167. http://dx.doi.org/10.1007/ s10750-006-0055-x

MANLY, BJF., 2008. Métodos estatísticos multivariados: uma introdução. 3. ed. Tradução de S. I. Carmona. Porto Alegre: Bookman. 
MAGURRAN, AE., 2004. Measuring biological diversity. Oxford: Blackwell Publishing Company.

MOOJEN, J., CARVALHO, JC. and LOPES, HS., 1941. Observações sobre o conteúdo gástrico das aves brasileiras. Memórias do Instituto Oswaldo Cruz, vol. 36, no. 3, p. 405-444. http://dx.doi. org/10.1590/S0074-02761941000300016

MOTTA-JUNIOR, JC., 1990. Estrutura trófica e composição das avifaunas de três habitats terrestres na região central do estado de São Paulo. Ararajuba, vol. 1, p. 65-71.

MOTTA-JUNIOR, JC., GRANZINOLLI, MAM. and DEVELEY, PF., 2008. Aves da estação ecológica de Itirapina, estado de São Paulo, Brasil. Biota Neotropica, vol. 8, no. 3, p. 207-227.

MURAKAMI, M. and NAKANO, S., 2002. Indirect effect of aquatic insect emergence on a terrestrial insect population through predation by birds. Ecology Letters, vol. 5, p. 333-337. http:// dx.doi.org/10.1046/j.1461-0248.2002.00321.x

MYERS, N., MITTERMEIER, RA., MITTERMEIER, CG., FONSECA GAB. and KENT, J., 2000. Biodiversity hotspots for conservation priorities. Nature, vol. 403, p. 853-858. http:// dx.doi.org/10.1038/35002501

O'CONNEL, TJ., JACKSON, LE. and BROOKS, RP., 2000. Bird guilds as indicators of ecological condition in the central Appalachians. Ecological Applications, vol. 10, no. 6, p. 1706-1721. http://dx.doi.org/10.1890/1051-0761(2000)010[1706:BGAIO E]2.0.CO;2

OLMOS, F., 2005. Aves ameaçadas, prioridades e políticas de conservação no Brasil. Natureza \& Conservação, vol. 3, no. 1, p. $21-42$.

POWER, ME. and RAINEY. WE., 2000. Food webs and resource sheds: towards spatially delimiting trophic interactions. In HUTCHINGS, MJ., JOHN, EA. and STEWART, AJA. The ecological consequences of environmental heterogeneity. Cambridge: Blackwell Science.

RATTER, JA., RIBEIRO, JF. and BRIDGEWATER, S., 1997. The Brazilian cerrado vegetation and threats to its biodiversity. Annals of Botany, vol. 80, p. 223-230. http://dx.doi.org/10.1006/ anbo.1997.0469

REIS, MG., 2011. Ecologia de bandos mistos de aves na Estação Ecológica de Itirapina, estado de São Paulo. São Carlos: Universidade Fedetal de São Carlos. Dissertação de Mestrado.

RIBEIRO, JF. and WALTER, BMT., 1998. Fitofisionomias do Bioma Cerrado. In SANO, SM. and ALMEIDA, SP. (Eds.). Cerrado: ambiente e flora. Embrapa/CPAC: Brasília. p. 89-166.

RICKLEFS, RE., 2003. A Economia da Natureza. 5. ed. Rio de Janeiro: Guanabara Koogan.

RICKLEFS, RE. and HAINSWORTH, FR., 1968. Temperature Dependent Behavior of the Cactus Wren. Ecology, vol. 49, no. 2, p. 227-233. http://dx.doi.org/10.2307/1934451

São Paulo. Secretaria do Meio Ambiente, 2009. Fauna ameaçada de extinção no estado de São Paulo: vertebrados. São Paulo: Fundação Parque Zoológico de São Paulo, Secretaria do Meio Ambiente.

SARMIENTO, G., 1983. The savannas of Tropical America. In BOURLIERE, F. Ecossystems of the world: Tropical Savannas. Amsterdam: Elsevier.

SCARIOT, A., SOUSA-SILVA, JC. and FELFILI, JM., 2005. Cerrado: Ecologia, Biodiversidade e Conservação. Brasília: Ministério do Meio Ambiente.
SICK, H.,1997. Ornitologia Brasileira. Rio de Janeiro: Ed. Nova Fronteira. Edição revista e ampliada por J.F. PACHECO, 2001.

SIGRIST, T., 2006. Aves do Brasil: uma visão artística. 2. ed. São Paulo: Editora Avis Brasilis.

-, 2009. Iconografia das aves do Brasil: Bioma Cerrado. Vinhedo: Avis Brasilis. vol. 1.

SILVA, DA., 2005. Levantamento do meio físico das Estações Ecológica e Experimental de Itirapina, São Paulo, Brasil. Revista do Instituto Florestal, vol. 17, no. 1, p. 113-128.

SILVA, JMC., 1997. Endemic bird species and conservation in the Cerrado region, South America. Biodiversity and Conservation, vol. 6, p. 435-450. http://dx.doi.org/10.1023/A:1018368809116

SILVA, JMC. and BATES, JM., 2002. Biogeographic patterns and conservation in South American cerrado: a tropical savanna hotspot. BioScience, vol. 52, p. 225-233. http://dx.doi.org/10.1641/00063568(2002)052[0225:BPACIT]2.0.CO;2

SILVA, WR. and GIARETTA, AA., 2008. Further notes on the natural history of the South American pepper frog, Leptodactylus labyrinthicus (Spix, 1824) (Anura, Leptodactylidae). Brazilian Journal of Biology, vol. 68, no. 2, p. 403-407. http://dx.doi. org/10.1590/S1519-69842008000200024

SIMBERLOFF, D. and DAYAN, T., 1991. The Guild concept and the structure of ecological communities. Annual Review of Ecology, Evolution and Systematics, vol. 22, p. 115-143. http:// dx.doi.org/10.1146/annurev.es.22.110191.000555

STOTZ, DF., FITZPATRICK JW., PARKER III, TA. and MOSKOVITS, DK., 1996. Neotropical Birds: Ecology and Conservation. University of Chicago Press.

TANNUS, JLS., 2007. Estudo da vegetação dos campos úmidos de cerrado: aspectos florísticos e ecológicos. São Paulo: Universidade de São Paulo. Tese de doutorado.

TANNUS, JLS., ASSIS, MA., and MORELLATO, PC., 2006. Fenologia reprodutiva em campo sujo e campo úmido numa área de cerrado no sudeste do Brasil, Itirapina - SP. Biota Neotropica, vol. 6 , no. 3 .

TELINO JUNIOR, WR., AZEVEDO JUNIOR, SM. and NEVES, RML., 2003. Biologia e censo de Porphyrula martinica, Gallinula chloropus e Jacana jacana em Dois Irmãos, Pernambuco, Brasil. Lundiana, vol. 4, no. 1, p. 43-49.

VICKERY, PD., TUBARO, PL., SILVA, JMC., PETERJOHN, BG., HERKERT, JR. and CAVALCANTI, RB., 1999. Conservation of grassland birds in the western hemisphere. Studies in Avian Biology, vol. 19, p. 2-26.

WILBUR, HM., 1997. Experimental ecology of food webs: complex systems in temporary ponds. Ecology, vol. 78, p. 22792302. http://dx.doi.org/10.1890/0012-9658(1997)078[2279:EE OFWC]2.0.CO;2

WILLIS, EO., 2004. Birds of a habitat spectrum in the Itirapina Savanna, São Paulo, Brazil (1982-2003). Brazilian Journal of Biology, vol. 64, p. 901-910.

YANG, LH., BASTOW,JL., SPENCE, KO. and WRIGHT, AN., 2008. What we can learn from resource pulses? Ecology, vol. 89 , no. 3, p. 631-634.

ZANCHETTA, D., SILVA, CEF., REIS, CM., SILVA, DA., LUCA, EF., FERNANDES, FS., LUTGENS, HD., TANNUS, JLS., PINHEIRO, LS., MARTINS, MRC. and SAWAYA, R., 2006. Plano de Manejo Integrado: Estações Ecológica e Experimental de Itirapina. São Paulo: Instituto Florestal.

ZAR, JH., 1999. Biostatistical analysis. 4. ed. Upper Saddle River: Prentice Hall. 
